\title{
Single cell transcriptional zonation of human psoriasis skin identifies an alternative immunoregulatory axis conducted by skin resident cells
}

\author{
Yuge Gao ${ }^{1,2}$, Xinyu Yao ${ }^{3}$, Yumeng Zhai ${ }^{1}, \mathrm{Li} \mathrm{Li}^{4}$, Huini Li ${ }^{2}$, Xianqi Sun ${ }^{1}$, Pei Yu', Tiankuo Xue ${ }^{1}$, Yuzhen $\mathrm{Li}^{1}$ and
} Yizhou Hu (10 5

\begin{abstract}
Psoriasis is the most common skin disease in adults. Current experimental and clinical evidences suggested the infiltrating immune cells could target local skin cells and thus induce psoriatic phenotype. However, recent studies indicated the existence of a potential feedback signaling loop from local resident skin cells to infiltrating immune cells. Here, we deconstructed the full-thickness human skins of both healthy donors and patients with psoriasis vulgaris at single cell transcriptional level, and further built a neural-network classifier to evaluate the evolutional conservation of skin cell types between mouse and human. Last, we systematically evaluated the intrinsic and intercellular molecular alterations of each cell type between healthy and psoriatic skin. Cross-checking with psoriasis susceptibility gene loci, cell-type based differential expression, and ligand-receptor communication revealed that the resident psoriatic skin cells including mesenchymal and epidermis cell types, which specifically harbored the target genes of psoriasis susceptibility loci, intensively evoked the expression of major histocompatibility complex (MHC) genes, upregulated interferon (INF), tumor necrosis factor (TNF) signalling and increased cytokine gene expression for primarily aiming the neighboring dendritic cells in psoriasis. The comprehensive exploration and pathological observation of psoriasis patient biopsies proposed an uncovered immunoregulatory axis from skin local resident cells to immune cells, thus provided a novel insight for psoriasis treatment. In addition, we published a user-friendly website to exhibit the transcriptional change of each cell type between healthy and psoriatic human skin.
\end{abstract}

\section{Introduction}

Psoriasis is the most common chronic, immunemediated disorder skin disease in adults, with an estimated global prevalence of $2-3 \%^{1}$, of which $90 \%$ is psoriasis vulgaris ${ }^{2}$. The pathophysiology of psoriasis

\footnotetext{
Correspondence: Yuzhen Li (lyzdermatology@163.com) or Yizhou Hu (yizhou.hu@ki.se)

'Department of Dermatology, The Second Affiliated Hospital of Harbin Medical University, No.246 Xuefu Road, 150001 Harbin, China

${ }^{2}$ Institute of Biotechnology, HILIFE Unit, University of Helsinki, 00014 Helsinki, Finland

Full list of author information is available at the end of the article

These authors contributed equally: Yuzhen Li, Yizhou Hu

Edited by D. Aberdam
}

vulgaris is characterized by abnormal keratinocyte proliferation and immune cell infiltration in the dermis and epidermis, further affect the overall innate and adaptive immune systems ${ }^{3}$. Therefore, psoriasis vulgaris and its comorbidities have caused serious physical and mental burden on patients all over the world ${ }^{2,4-6}$.

Even though psoriasis is one of the most studied dermatological diseases, its pathogenesis is still not completely elucidated. Accumulating cellular and molecular regulators have been found to be pivotal during the pathogenesis of psoriasis vulgaris. Previous experimental and clinical evidences have established the inflammatory roadmap from infiltrating immune cells including

\section{(c) The Author(s) 2021}

(c) (i) Open Access This article is licensed under a Creative Commons Attribution 4.0 International License, which permits use, sharing, adaptation, distribution and reproduction cc) in any medium or format, as long as you give appropriate credit to the original author(s) and the source, provide a link to the Creative Commons license, and indicate if changes were made. The images or other third party material in this article are included in the article's Creative Commons license, unless indicated otherwise in a credit line to the material. If material is not included in the article's Creative Commons license and your intended use is not permitted by statutory regulation or exceeds the permitted use, you will need to obtain permission directly from the copyright holder. To view a copy of this license, visit http://creativecommons.org/licenses/by/4.0/. 
neutrophils, $\mathrm{T}$ cells, dendritic cells (DCs) to skin resident cells, especially the keratinocytes ${ }^{2,3,7}$. Most of these circuits are directly or indirectly mediated by chemokines and cytokines. Current knowledge indicated that multiple factors could induce the activation of myeloid dendritic cells (mDCs) and the production of interleukin (IL)-23, which in turn stimulated the major $\mathrm{T}$ cell subsets to secrete IL-17 (ref. ${ }^{8,9}$ ). This pathogenic model of psoriasis vulgaris based on the IL-23/IL-17 axis is widely accepted $^{8,10,11}$. Likewise, both IL-17 and IL-23 inhibitors have shown good efficacy and safety in the systemic treatment of psoriasis. However, these inhibitors could not completely alleviate the disease ${ }^{12}$, suggesting alternative regulatory events of psoriasis beyond IL-23/IL-17 signaling. Recent studies revealed a few molecular feedback events occurred from the skin resident cells ${ }^{13,14}$, indicating a potential indirect immune regulatory axis driven by the resident cells. However, previous studies mostly either focused on one solitary signaling axis or profiled the genetic alterations of human psoriasis skin at the bulk tissue level ${ }^{15-17}$, which led to the obscuration of the overall contribution of psoriasis-related genetic characteristics to specific cell type. Therefore, urgently exploration is needed to systematically study the inherent transcriptional changes and intercellular molecular communication of each specific cell type in human psoriasis full-thickness skin.

In this study, we used single-cell RNA sequencing (scRNAseq) to deconvolute a high-quality transcriptional landscape of human epidermal and dermal skin cells from both healthy donors and patients with psoriasis vulgaris. Through the combination of neural-network learning, multilayer analysis of genome/transcriptome/ligand-receptor and pathological investigation of patient biopsies, we further revealed the evolutionally conserved skin resident cells, including both epidermal keratinocytes and dermal mesenchymal cells, could self-transform into immune active status by evoking MHC genes during psoriasis vulgaris, and further nutrient immune cells via upregulating cytokine genes and extracellular matrix related genes.

Last, we built a user-friendly website for exploring the gene expression of normal/psoriasis human skin cells: https://yzstudio.one/skin-psoriasis-atlas.

\section{Result}

\section{Single cell transcriptional profiling of the full-thickness healthy and psoriatic skin}

To systematically and comprehensively address the complexity and potential pathological change of cell types in both dermis and epidermis of patients with psoriasis vulgaris, single cells from full-thickness skins were collected from the lesion region of three patients (two male and one female) and the similar region of three healthy donors (two male and one female). All tissues were subsequently profiled with single cell RNA sequencing (Fig.
1A; SI Table 1). After enzymatically and mechanically disrupting the tissue, single cell suspension (viability $>90 \%$ ) was subjected to scRNAseq. 24,259 single cells were passed the initial quality control with the median detected UMI reads of 8858 and median detected proteincoding genes of 2747 , mitochondrial gene ratio $<5 \%$. And the doublets (estimated ratio $\sim 3.3 \%$ ) were removed (SI Fig. 1A-D). In addition, we observed one patient harbored HLA-C*06:02, a primary susceptibility allele for psoriasis $^{18,19}$ (SI Fig. 1E).

All 24,259 cells were further clustered to five main clusters and one erythrocyte cluster $\left(\mathrm{HBA}^{+} \mathrm{HBB}^{+}\right.$ $\mathrm{HBA}^{+}$cells). After excluding erythrocyte cluster (25 cells), the rest five main clusters of 24,234 cells were further clustered into 35 sub-cell types and visualized in a uniform manifold approximation and projection (UMAP) plot (Fig. 1B, left). Each cell-type contained cells from all investigated donors as visualized in (Fig. 1B, right), but cell-type composition changes of psoriatic and healthy cells varied among some cell types, as described in (Fig. 3A, B). After comparing well known markers with the most representative expressed gene markers of each cluster, five main clusters were defined as follows: endothelial (End/Endo), majorly expressing CDH5, PECAM1, CD34; epidermis (EpD), majorly expressing KRT10, KRT14, KRT1; immune (IM), majorly expressing PTPRC (CD45), ITGAM, CD3E; mesenchymal (Mes), majorly expressing PDGFRB, LUM, COL1A1; neural crest-like (Schwann/Melanocyte-like, SchM), majorly expressing SOX10, MLANA, S100B (Fig. 1C).

As the most abundant skin cell type, EpD accounted for $37.96 \%$ (9201 cells) of all skin cells, which could be divided into nine different clusters, including three basal cell types (EpD_Basal1-3) majorly expressing basal epithelium markers KRT5, KRT14, ITGA6, and ITGB1; four epithelial differentiated cell types (EpD_Spinous, EpD_Granualr/spinous, EpD_Granular, EpD_Corneum) majorly recognized by epithelial differentiation marker KRT10, KRT1, and GRHL1 (ref. ${ }^{20-23}$ ); sebaceous gland (EpD_SG) expressing APOC1, ACSL5, ABCC3 (ref. ${ }^{24}$ ); follicles (EpD_Foli) expressing CD200, SOX9, KRT19 (ref. ${ }^{25,26}$ ). End/Endo constituted $15.69 \%$ (3802 cells) of the full-thickness skin cells, was identified by its archetypal marker PECAM1, which was represented by four endothelial cell types (End_Endo1-4) and a lymphatic endothelial cell type (Endo_lym) ${ }^{27}$. In total, 7642 mesenchymal cells counted for $31.53 \%$ of the fullthickness skin cells were represented by six clusters included fibroblast (Mes_Fibro), 2 fibroblast/pericyteslike cell types (Mes_Peri1-2), two dermal sheath/dermal papilla cell types (Mes_DS/DP_1-2), and smooth musclelike (Mes_SM). We detected 2983 immune cells, 12.3\% of the total cells, and divided them into 10 clusters, namely three T cell types (IM_CD4+, IM_CD8+, IM_Treg), two 
(A)

$3 \sqrt{3} \rightarrow$

Psoriatic/healthy donors

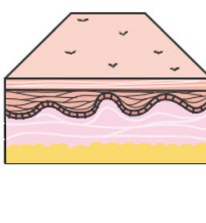

Full-Thickness samples
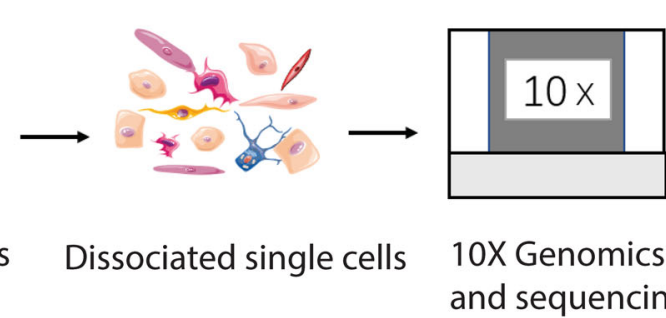

10X Genomics and sequencing

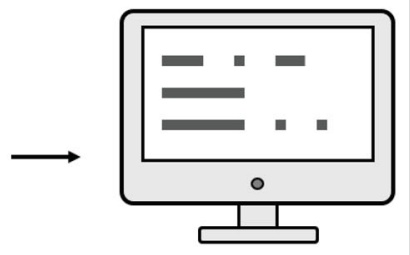

Data Analysis

(B)

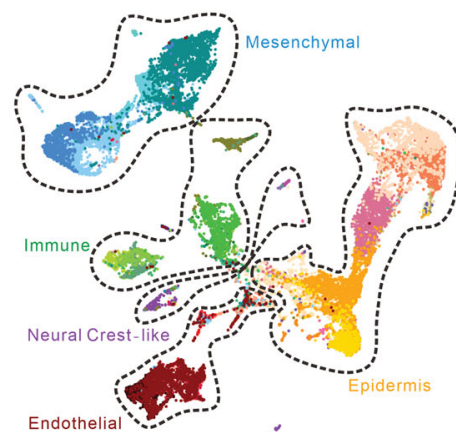

(C)

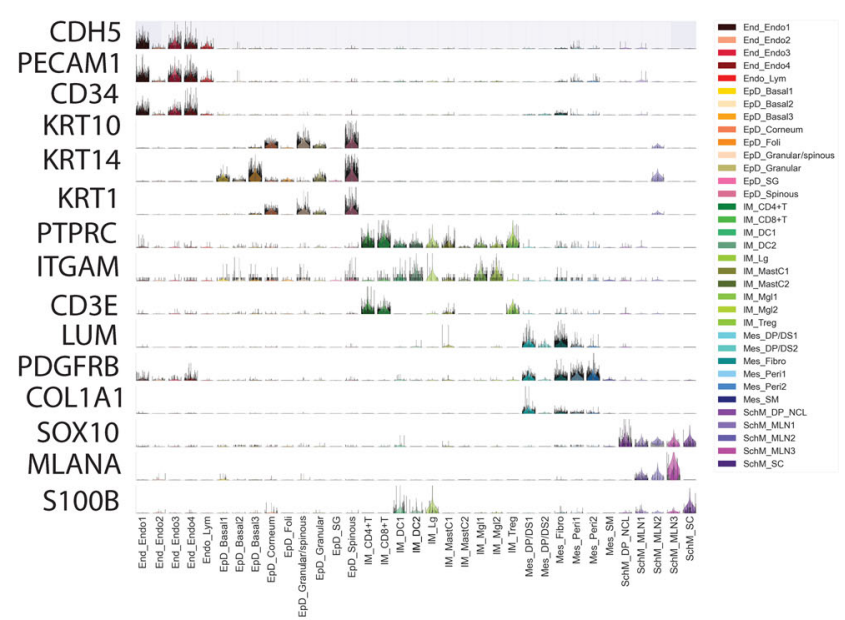

(D)

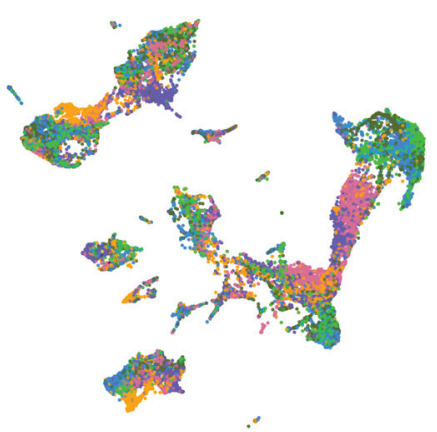

Ctr1 $=$ Psor1
Ctr12 Psor2 Ctrl3 Psor2
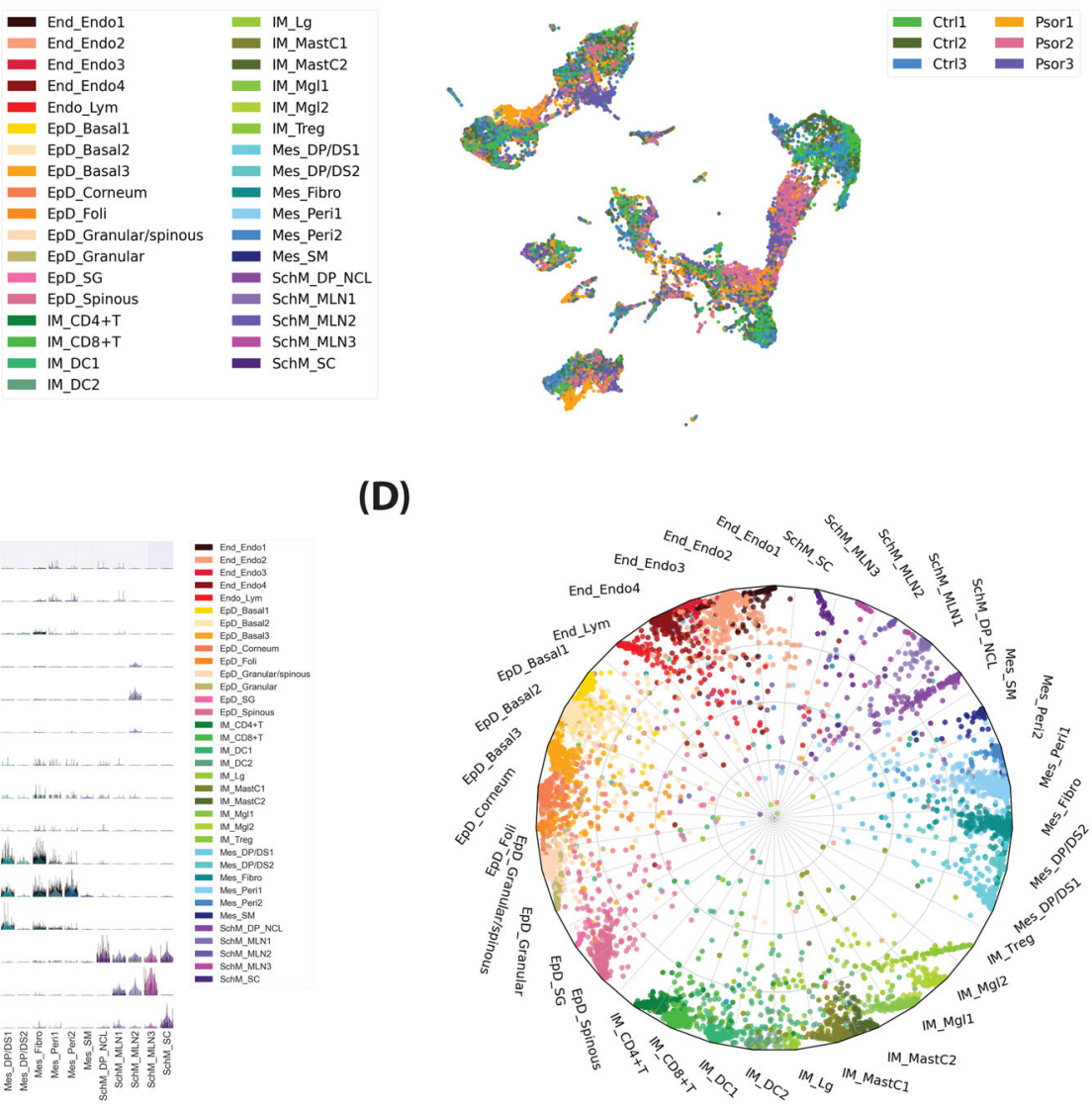

Fig. 1 Clustering, marker genes and cell type assignment of human full-thickness skin scRNAseq from healthy and psoriatic donors.

A Schematic view of the workflow. B Left, human full-thickness skin cell types. Visualization using UMAP of skin cells, each point represents one cell. Color coding based on unique cell clusters, as shown at right beside. Right, UMAP plot was folked from left side and colored by samples. Ctrl 1-3, samples from healthy donors; Psor 1-3, samples from patients with psoriasis vulgaris. C Expression of selected top significant marker genes for five main cell type clusters; relative expression of each gene in each cluster was visualized as violin plot, and the raw counts of each gene were visualized as sparkline plot in that violin plot. D Radar plot visualization of cell-type probabilistic scores of human full-thickness skin cells in relation to our defined cell types. Each dot represented one cell. Color coding based on unique cell clusters. The position of each dot indicated the cell-type score between that cell and the training (defined) cell types which were indicated outside of each bend in the radar wheel. Almost all cells were correctly assigned to their defined cell types. 
dendritic cell types (IM_DC1-2), Langerhans (IM_Lg), two mast cells (IM_MastC1-2), and two macrophage-like cells (IM_Mgl1-2). We detected 606 SOX10+ cells as SchM neural crest-related cells, occupying $2.5 \%$ of all skin cells, including three melanocyte clusters (SchM_MLN1-3), a dermal papilla cell/neural crest-like cluster (SchM_DP_NCL), and one Schwann cell cluster (SchM_SC). Each cluster had unique transcriptional markers (SI Table 2a), and was in line with previous known markers in that type of cells (SI Table $2 b)^{28-30}$, selected marker genes were visualized in (SI Fig. 1E). To further validate the accuracy of our classification, we applied cross-validation driven by a vanilla neuralnetwork. The classifier successfully assigned each cell into their defined cluster (Fig. 1D) with an accuracy near 80\% (SI Fig. 1F, G). Randomized expression matrix was used as negative control, and in the same model, none of randomized cells significantly assigned to the right cluster (SI Fig. 1H), suggesting the robustness of our classification. The expression of all protein-coding gene of each cluster was visualized as violin-plot and UMAP plot in the webpage: https://yzstudio.one/skin-psoriasis-atlas.

\section{The anatomical location and evolutional conversation of human skin cell type}

To explore the anatomical regions of our defined cell types, we compared our data with published human skin scRNAseq data only from epidermis layer ${ }^{31}$ or from fullthickness $\operatorname{skin}^{32}$. To exert the cell-type comparison at single cell resolution, we applied previously built neuralnetwork classifier (Fig. 1D), which was used the trained knowledge-supervised cell-type as machine learning model to evaluate the weighted gene-pattern relations and then to systematically quantify the probabilistic similarity of each predicted cell against our defined cell types. Therefore, the cell-to-cell type similarity did not rely on a few marker genes, but the comprehensively weighted gene patterns, and thus significantly enhanced the evaluation accuracy. We successfully assigned epidermis skin cells (Fig. 2A) and full-thickness skin cells (Fig. 2B) into our clusters, as visualized in radar plot. Overall, most of the predicted full-thickness skin cells were assigned to our defined cell types (Fig. 2B), and all our defined cell types contained the predicted full-thickness skin cells, indicating that our defined cell types could correctly classify and represent the full-thickness skin cells from other published data. In contrast, all epidermal skin cells were only assigned to some of our defined cell types, including all epidermis clusters, most immune cell types except IM_MastC2, and two types of melanocytes (SchM_MLN1, SchM_MLN3). Compared with the assignment of fullthickness skin data, we confidently identified the cell types specifically at skin dermis or the junction region of epidermis/dermis, including all endothelial cells, all mesenchymal cells, IM_MastC2 mast cells, a type of melanocyte (SchM_MLN2), dermal papilla cell/neural crest-like cluster (SchM_DP_NCL), and Schwann cells (SchM_SC), as indicated with red frame in (Fig. 2C). This finding was in line with previous studies that most blood vessel cells and mesenchymal cells located in the skin dermis $^{29,32}$, and most of the human melanocytes located among keratinocytes in the epidermis layer or junction region $^{33}$, while Schwann precursor cells had been collected from adult human dermis ${ }^{34,35}$.

Next, we evaluated the evolutional conservation of human and mouse skin cell types. The same neuralnetwork classifier was applied to compare our human cell types with published mouse scRNAseq data from mouse skin $^{30}$ or psoriatic mouse $\operatorname{skin}^{36}$. Among five main cell types, three main types including epidermis, endothelial, and mesenchymal were relatively evolutional conserved with the minor inconsistence that mouse skin harbored much less epidermis granular cells (EpD_Granular), epidermis hair follicle cells (EpD_Foli), epidermis sebaceous gland cells (EpD_SG), despite the different experimental protocols and capture efficiencies (Fig. 2D; SI Fig. 2). We observed in this dataset that psoriatic mouse skin had significant high ratio of different types of immune cells than normal mouse skin (Fig. 2E), in line with the observation of infiltrating immune cells in skin region during psoriasis. Moreover, all the immune cell types were also similar to human skin except Treg cells. In contrast to the four main types above, most $\mathrm{SOX}^{+} 0^{+}$cell types (SchM main types) exhibited significantly evolutional insistence, although the major trend of similarity was observed. None of neural crest-like cells of mouse skin was exactly assigned to human $\mathrm{SOX} 10^{+}$cell types, except a few of psoriatic mouse skin cells were assigned to human melanocytes (SchM_MLN3) and human Schwann cells (SchM_SC).

\section{Skin resident cell types exhibited immune regulatory ability}

We further analyzed the cell-type distribution of different individuals, including 11,417 cells from three healthy donors and 12,817 cells from three donors with psoriasis, and observed the changes of cell-type composition between psoriasis and the health. Statistical analysis revealed that EpD_Corneum, SchM_MLN1, EpD_Granular/spinous, EpD_Basal1, EpD_SG, SchM_MLN3, EpD_Granular, IM_MastC1 cells showed a significant decreasing in psoriasis, while EpD_Spinous, End_Endo3, EpD_Basal3, IM_Mgl2, SchM_DP_NCL, IM_MastC2, Mes_DP/DS1, End_Endo2, Mes_Peri1 cells increased significantly (Fig. 3A, B).

The cell-type composition changes largely reflected cell viability (autonomous) and motility (non-autonomous), and indicated the cell-based functional shift in this region. 
(A)

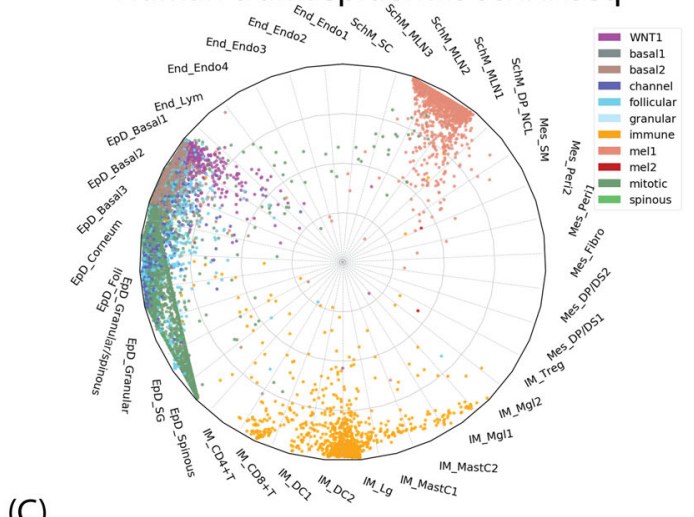

(B)

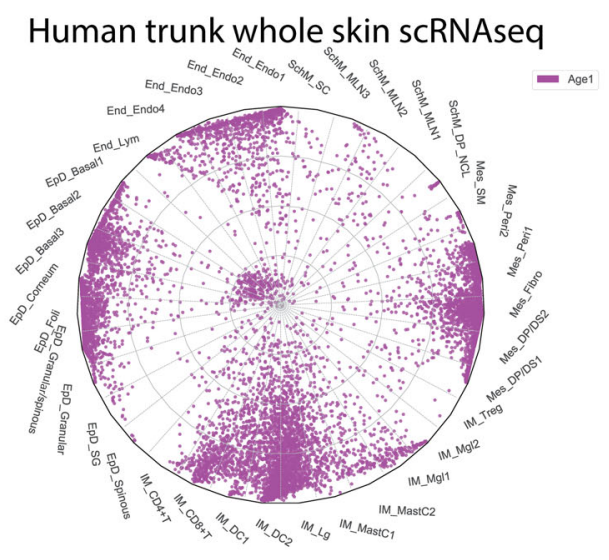

(C)

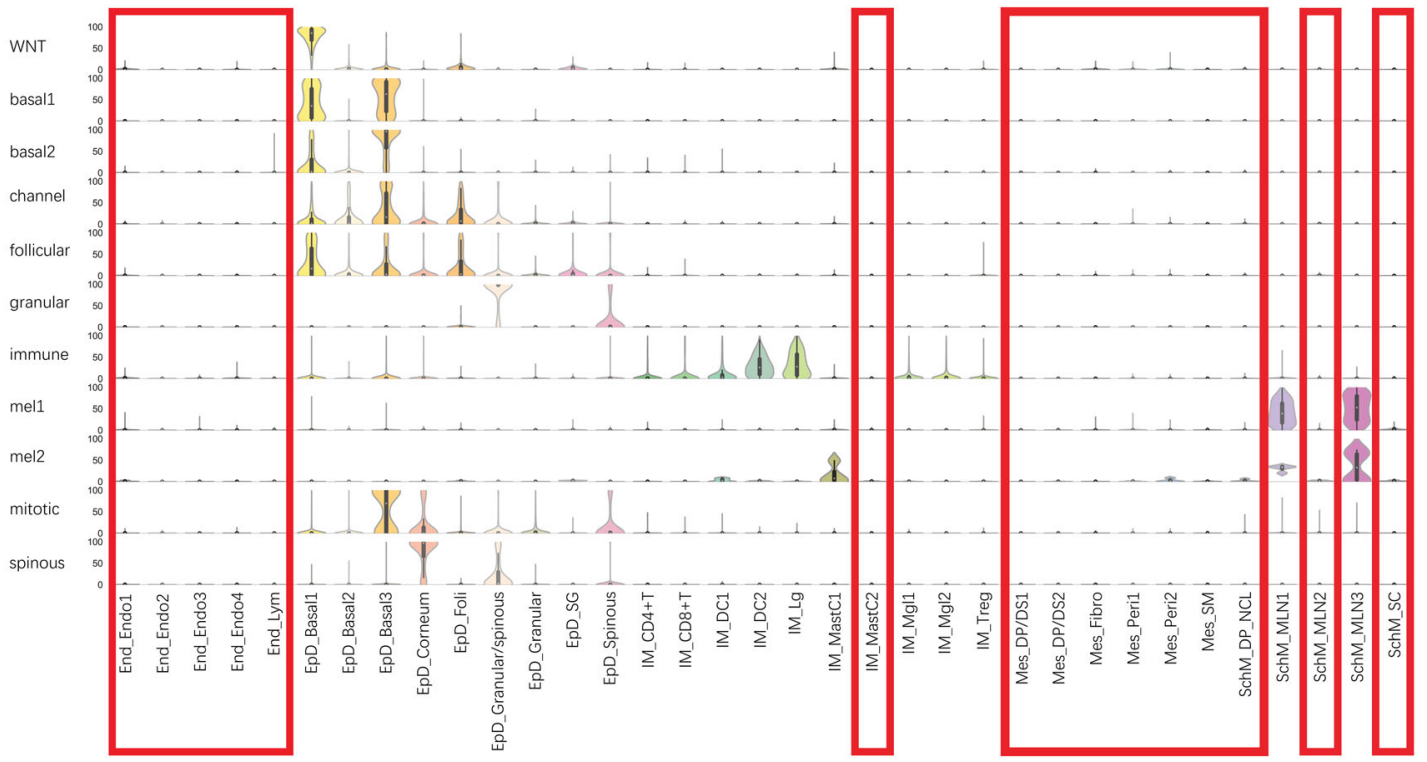

(D)

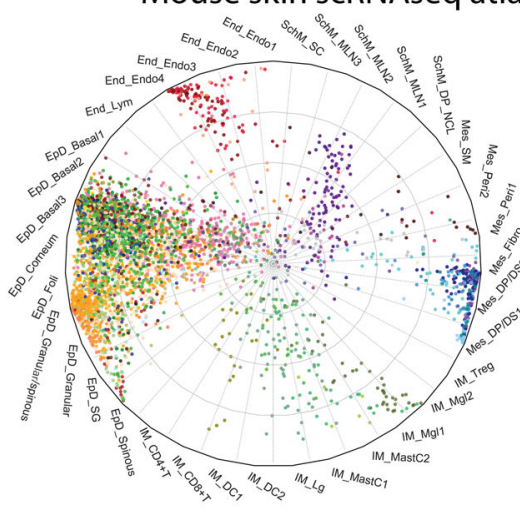

(E)
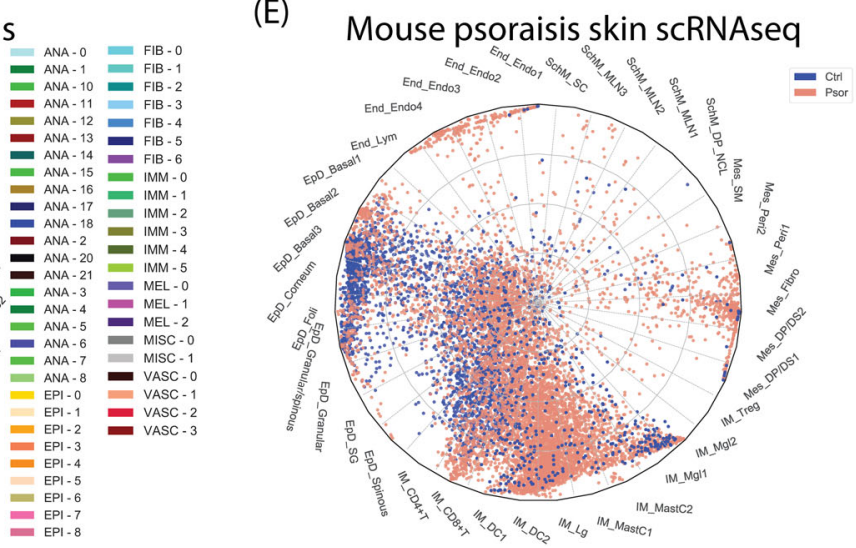

Fig. 2 Prediction of the anatomical location and evolutional conversation of human skin cell type. A Radar plot visualization of cell-type probabilistic scores of published human trunk epidermis skin cells in relation to our defined cell types. B Radar plot visualization of cell-type probabilistic scores of published human full-thickness skin cells in relation to our defined cell types. C Violin plot visualization of cell-type probabilistic scores of published human trunk epidermal skin cells in relation to our defined cell types. $X$-axis represented our defined cell types, $y$-axis represented defined cell types from original study. Red frame indicated low similar (absent) cell types of our defined cell types in epidermal skin cell types. D Radar plot visualization of cell-type probabilistic scores of published mouse skin atlas cells in relation to our defined human skin cell types. E Radar plot visualization of cell-type probabilistic scores of published mice healthy or psoriatic skin cells in relation to our defined human skin cell types. For all radar plots, each dot represented one cell. Color coding based on their original defined cell clusters or disease condition. The position of each dot indicated the cell-type score between that cell and the training (defined) cell types which were indicated outside of each bend in the radar wheel. 
(A)

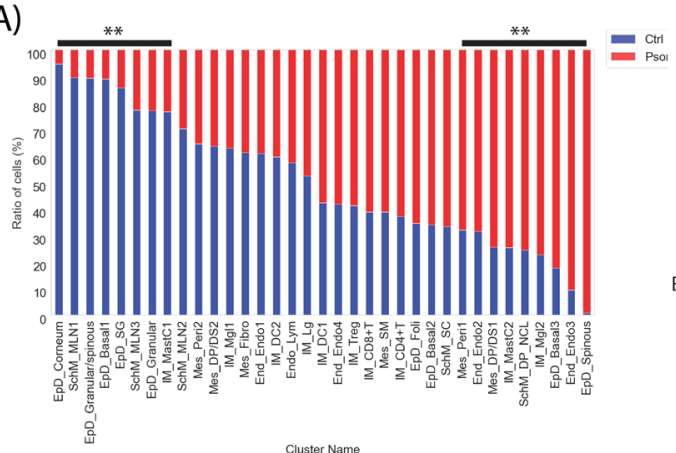

(C)

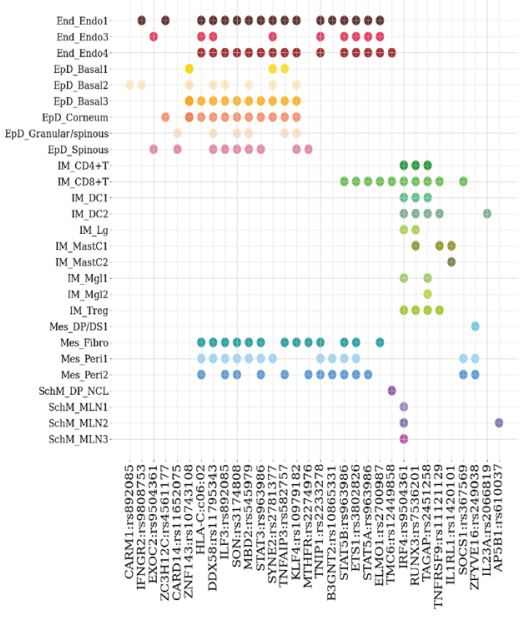

(E)

\section{Cluster EpD_Granular/spinous:}

(B)

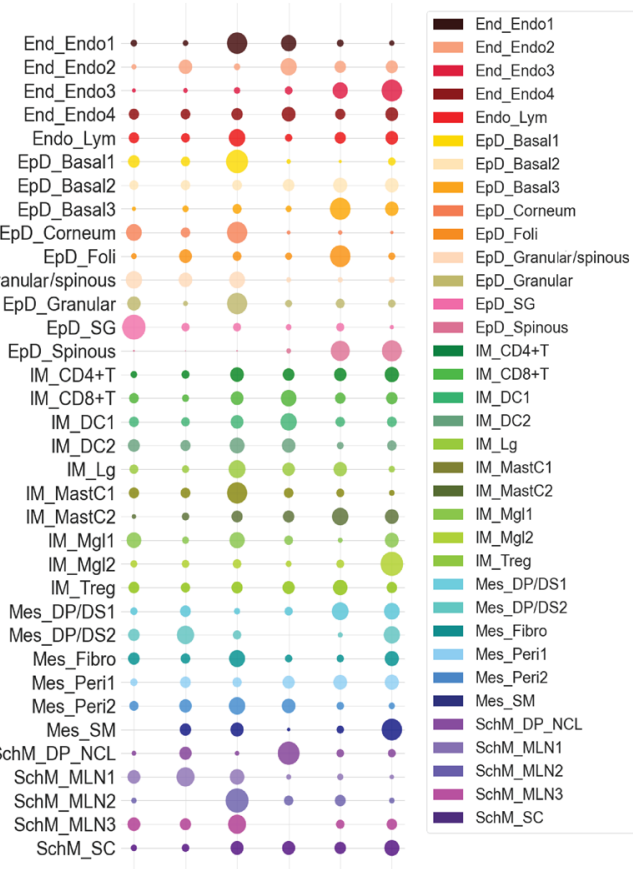

(D)

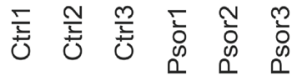
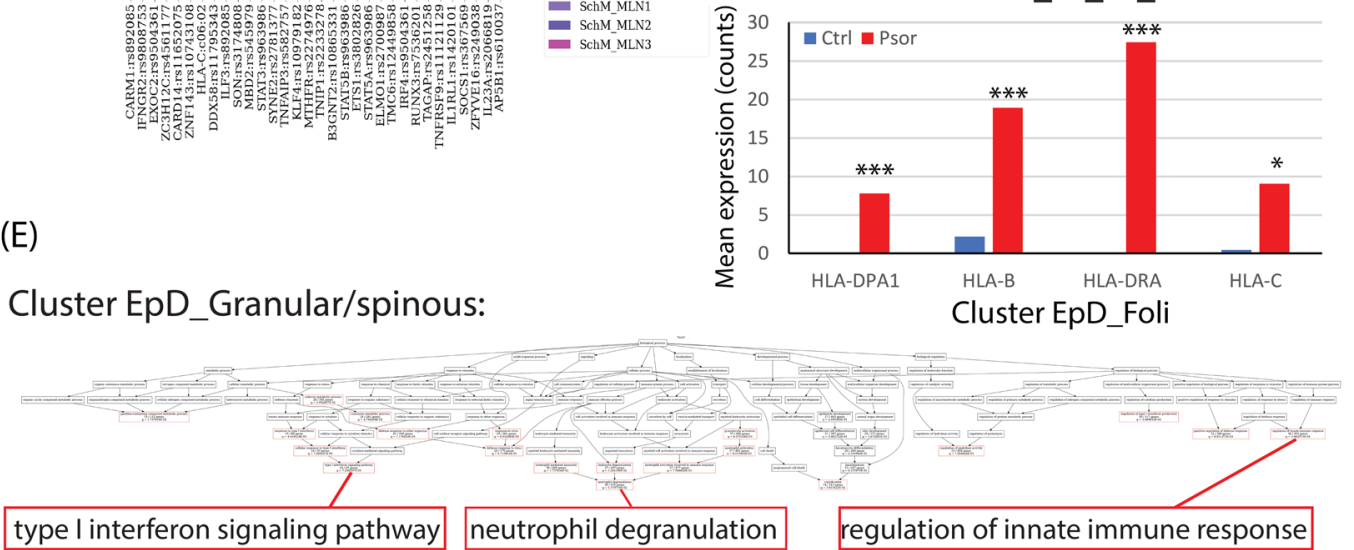

(F)

\section{Cluster Mes_Fibro:}

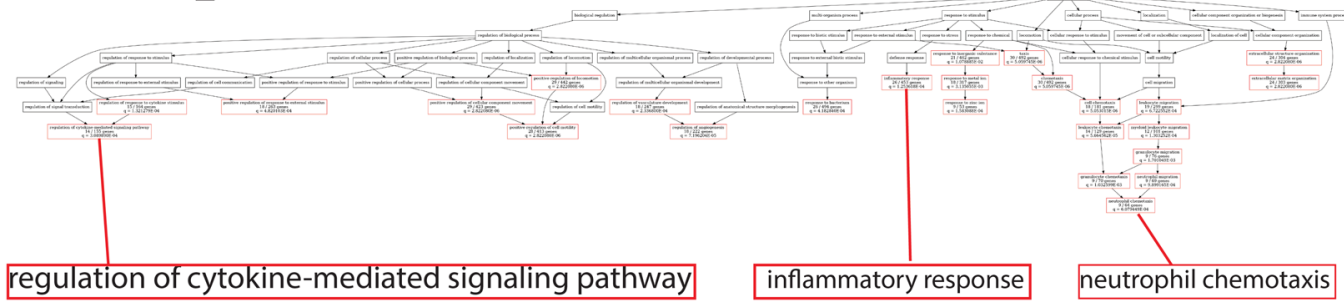

Fig. 3 The difference of cellular composition and cell-type-based transcription in psoriasis. A Percentage bar plot of cell type composition in healthy and psoriatic skin, $y$-axis represented the percent of total cell number of that cell type. Red color represented psoriatic skin and blue color represented healthy control skin. Significant changes (FDR $<0.01$ ) were labeled as ** on the top. B Dot plot of cell number distribution of each cell type in each healthy and psoriasis donor. Dot size represented the cell number percent of that donor ( $x$-axis) in that cell type (y-axis). Color indicated the cell types. C Dot plot of the significant existence of each susceptibility gene loci ( $x$-axis) in each cell type ( $y$-axis). Color indicated each cell type. D Bar plot of the raw counted of four selected HLA genes in cell type EpD_Foli. Red color represented psoriatic skin and blue color represents healthy control skin. Y-axis represents the mean value of the expression raw counts. ${ }^{* *}$, FDR $p<0.001 ;{ }^{*}, p<0.05$. E, F GO-bioprocess enrichment plot of all psoriasis upregulated genes in cell type EpD_Granular/spinous (E) and Mes_Fibro (F). Red frame highlighted the significant enriched GO terms and the terminally enriched GO-bioprocess were enlarged at the bottom of each plot. 
We further zoomed into the molecular changes of each cell type during psoriasis, which included three aspects: first, cell type assignment of psoriasis susceptibility gene loci; second, the differentially expressed genes among each cell type induced by psoriasis; last, the reorganized ligand-receptor interaction among each cell type.

First, we collected 45 experimentally identified psoriasis susceptibility gene loci and their target genes, including the primary susceptibility gene loci and HLA-C*06:02 (SI Table 3$)^{37,38}$. The cell types significantly expressing these genes were the most vulnerable cellular targets of the psoriasis genetic predisposition. After cross-checking of both 45 susceptibility gene loci and our cell-type marker genes. We identified 32 common psoriasis susceptibility gene loci, which were differentially distributed in 35 cell types of skin (Fig. 3C), the gene expression of each cell type could be searched in our webpage: https://yzstudio. one/skin-psoriasis-atlas. Most of these loci were related to immune regulation, especially IFN and TNF signaling, such as IFNGR2, IL23A, STAT3, STAT5, TNFAIP3, and so on. Interestingly, 24 out of 32 target genes were highly or shared expressed in local resident skin cells, especially in epidermal cells and mesenchymal cells, including CARM1, IFNGR2, EXOC2, ZC3H12C, CARD14, ZNF143, DDX58, HLA-C, ILF3, SON, MBD2, STAT3, SYNE2, TNFAIP3, KLF4, MTHFR, TNIP1, B3GNT2, STAT5B, ETS1, STAT5A, ELMO1, SOCS1, ZFYVE16. This result indicated that the psoriasis genetic predisposition could not only target commonly known immune cells, but also perturbate local resident skin cells including both epidermis cells and mesenchymal cells, with endorsing immuno-regulatory potential.

Next, we compared the differential gene expression of each cell type between healthy and psoriatic human skin. Statistical analysis enriched both upregulated and downregulated genes of each cell type in psoriatic human skin. Surprisingly, some local resident skin cell types significantly upregulated MHC complex molecules in psoriatic condition: epidermal cell type EpD_Basal2, EpD_Basal3, EpD_Foli highly expressed MHC-II molecules such as HLA-DRA, HLA-DMA, HLA-C; mesenchymal cell types Mes_DP/DS1, Mes_Fibro, Mes_Peri1, Mes_Peri2 highly expressed both MHC-I and MHC-II molecules such as HLA-A, HLA-B, HLA-C, HLA-DRA. Besides, dermal papilla cell/neural crest-like cells (SchM_DP_NCL) and Schwann cells (SchM_SC) highly expressed MHC-I molecules such as HLA-A, HLA-B, HLA-C. An example of the upregulated expression of MHC complex genes in EpD_Foli was shown as bar chart in (Fig. 3D) and all differential expression data was listed in (SI Table 4) and searchable in our webpage: https:// yzstudio.one/skin-psoriasis-atlas.

Furthermore, gene ontology enrichment (GO) analysis of the significant upregulated genes of epidermis and mesenchymal cell types revealed two types of main GO bioprocesses: First, immune-responses including interferon signaling, neutrophil modulation, cytokine and chemokine production, and so on; Second, the regulations of cell cycle and cell apoptosis, which were majorly activated in epidermal basal cells (EpD_Basal1-3). Thus, the upregulated genes of local resident skin cells mainly contributed to immune modulation during psoriasis. Representative GO-bioprocess enrichment of cell type EpD_Granualar/spinous was shown in (Fig. 3E, F) and full enrichment figures and lists of each cell type were in (SI Fig. 3; SI Table 5).

\section{Ligand-receptor analysis revealed the regulatory potential from resident epidermal/mesenchymal cells to dendritic cells during psoriasis}

To explore the cell-cell interaction among each cell type of psoriatic skin, we applied ligand-receptor analysis for all upregulated ligand genes and relatively constant expressed receptor genes of each cell type during psoriasis. Full ligand-receptor interactions of psoriatic cell types were visualized in chord plot (Fig. 4A, SI Table 6), The overall interactions uncovered that significantly activated ligands and interactions were from epidermal cells (EpD_Basal2-3, EpD_Spinous), mesenchymal cells (Mes_Fibro, Mes_Peri1-2), and some endothelial cells. Further activity scoring revealed that the top scored ligand-receptor interactions were enriched on these six top ligand secretion resident cell types (EpD_Basal2-3, EpD_Spinous, Mes_Fibro, Mes_Peri1-2) shared a specific receptor immune cell type: type1 dendritic cell (IM_DC1) (Fig. 4B, SI Table 7). Checking the molecules underlying these interactions revealed that the activated ligands of psoriatic epidermal cells include IL-36G, WNT5A, CD58, and so on (SI Table 6), which all had been shown to modulate $\mathrm{DCs}^{39-41}$. The activated ligands of psoriatic mesenchymal cells included two main types: ECM related proteins, including SDK1, FGF7, COMP, COL5A3, COL1A1; cytokines or related proteins, including LIF, IL6, IL-17B, TNFRSF11B, CCL26, CCL19 (Fig. 4C). The inflammatory effects of LIF and IL-6 had been studied in mesenchymal cells ${ }^{42}$, and here we focused on IL-17B, an uncovered inflammatory ligand in mesenchymal cells and an IL-17 family member. We observed IL-17B coexpressed with PDGFRB + mesenchymal cells in skin dermis layer, and the skin tissue distribution of PDGFRB protein was consistent with the previous studies ${ }^{43}$. Furthermore, we performed immunohischemistry staining for both healthy and psoriatic skin biopsies, and quantitatively compared the expression levels of PDGFRB and IL-17B protein. We found that although the protein amount of PDGFRB in skin dermis didn't change (Fig. 4E), IL-17B protein was significantly upregulated in psoriatic dermis region (Fig. 4F). We further estimated the cytokine 


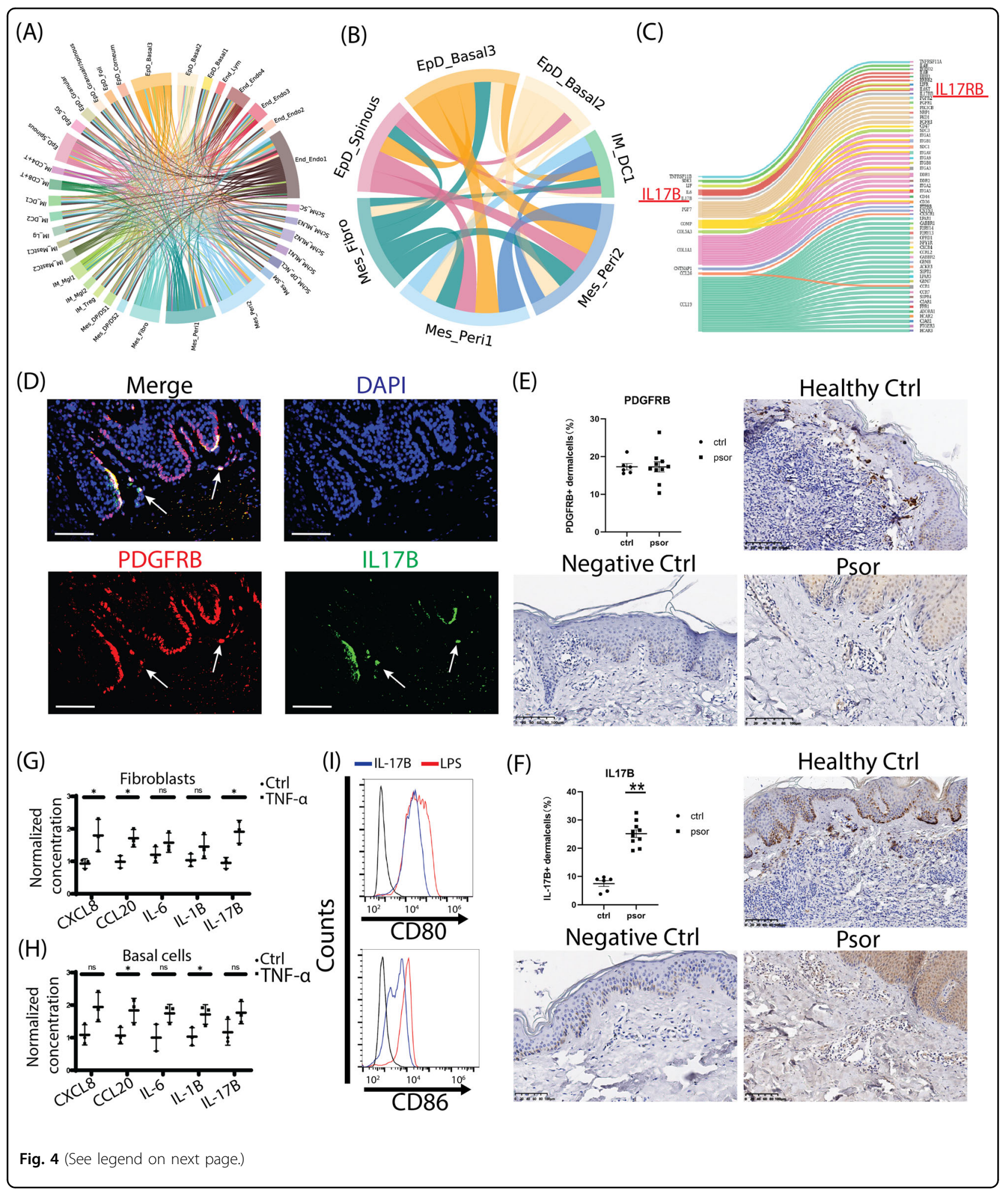

secretion of skin fibroblasts and basal cells under TNF- $\alpha$ stimulation, a canonical signaling involved in psoriasis and indicated by our susceptibility gene loci analysis (Fig. 3C). Upon TNF- $\alpha$ stimulation, CXCL8, CCL20, and IL17-
B significantly increased in fibroblasts (Fig. 4G), and the secretion of CCL20, IL-1B were enhanced by basal cells (Fig. 4H). Last, we observed that IL-17B contributed to the surface expression of CD80 and CD86 protein in DCs 
(see figure on previous page)

Fig. 4 Molecular interactions among cell types and the regulatory potentiality from epidermal/mesenchymal cells to dendritic cells during psoriasis. A Chord plot visualization of the ligand-receptor interactions among cell types. The defined cell types were indicated outside of each bend. Arc line between each cell types represented the ligand to receptor interactions, and the color indicated the ligand expressed cell types. B Chord plot visualization of the ligand-receptor interactions between top scored resident cell types and type 1 DC. The defined cell types were indicated outside of each bend. Arc line between each cell types represented the ligand to receptor interactions, and the colors indicated the ligand expressed cell types. C Sankey plot visualization of the interactions from the ligand of mesenchymal type cells to the receptors of type 1 DCs. The IL17B/LL-17RB pair was highlighted as red. D Immunofluorescence staining of the epidermis/dermis junction of human skins, PDGFRB protein was stained as red, IL-17B as green, and DAPI as blue, the cellular co-expressions of PDGFRB and IL-17B were observed at the dermis layer close to the junction (pointed by white arrow) and at the layer junction. Scale bar, $100 \mu \mathrm{M}$. E, F Immunohistochemistry staining and quantification of PDGFRB (E) and IL-17B (F) in healthy and psoriatic skins. Host IgG of primary antibodies were used as negative control. In dermis layer, the average PDGFRB protein amount didn't significantly change (E), but IL-17B protein significantly increased in psoriasis (F). Scale bar, $100 \mu \mathrm{M}$. Ctrl $N=6$; Psor $N=10$. ${ }^{* *}$, $p<0.01$. G, H The secreted protein levels of CXCL8, CCL20, IL-6, IL-1B, and IL-17B in skin fibroblasts (G) or skin basal cells (H) under TNF-a treatment were determined by ELISA. The protein concentrations were normalized to control group. $N \geq 3$; ${ }^{*}, p<0.05$; NS not significant. i Flow cytometry histograms of CD80 and CD86 expressions in DCs induced by IL-17B. LPS treatment was used as positive control.

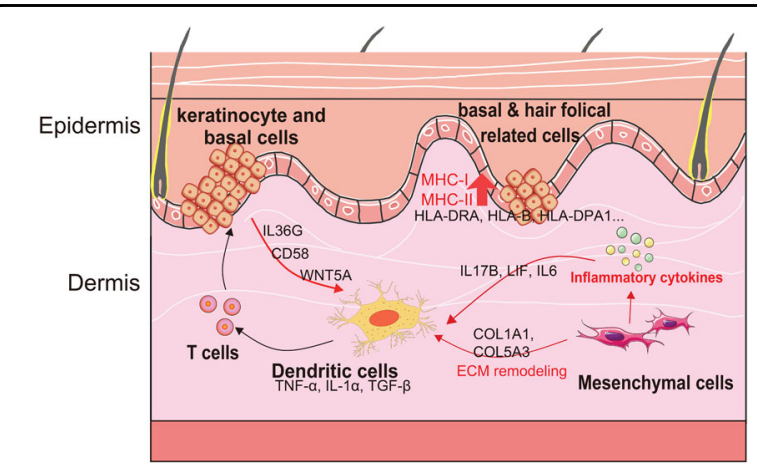

Fig. 5 Schematic view of the immune regulation from local resident epidermal cells and mesenchymal cells to DCs. Resident cells including basal cells, hair follicle-like cells, mesenchymal cells potentialize local inflammation by evoking MHC complex genes in psoriatic condition, and these cells can trigger dendritic cells for the inflammatory cascade through remodeling ECM and secreting cytokines such as LIF, IL-6, IL-17B, IL-36, and CD58.

(Fig. 4I). Thus, these results suggested that the enhanced secretion of IL-17B from dermal mesenchymal cells might actively contribute to the biological functions of immune cells during psoriasis.

\section{Discussion}

Single cell transcription technology has been used to study epidermal cells and immune cells in psoriatic $\operatorname{skin}^{31,44-46}$, but to our knowledge, our study is the first time that comprehensively deconstructs the resident cells from the full-thickness skin of human psoriasis at single cell transcriptional level (Fig. 5). The transcriptional markers of each cell type were mostly in line with previous knowledge (SI Table 2) and mouse skin scRNAseq studies $^{24,30,31,47}$. According to the transcriptional features of our defined human skin cell types, we trained a neuralnetwork learning model to automatically perform celltype assignment of any skin cells across species. Via this learning model, we further confirmed the evolutional conservation of most skin resident cell types between human and mouse. Furthermore, we found most human melanocytes and $\mathrm{SOX}_{10}{ }^{+}$dermal papilla-like cells, but not human Schwann cells exhibited evolutional specificities. These were probably due to the species difference of the environmental niche, where mouse melanocytes and dermal papilla cells majorly existed near the hair follicle region deeply into the dermis, while human cells usually rested among epidermis/dermis junction ${ }^{33}$.

We mapped the previously identified genomic loci implicated in psoriasis onto specific human skin cell types, and enriched 32 cell type specific genes targeted by the psoriasis genetic predisposition. Surprisingly, although most of these genes were originally interpreted as immune regulators, 24 out of 32 genes were significantly expressed in skin resident cells including keratinocytes or mesenchymal cells. And these 24 genes mainly contributed to IFN signaling and TNF signaling (such as IFNGR2, IL23A, STAT3, STAT5, TNFAIP3). This finding agreed with previous studies of experimental validation ${ }^{48-51}$, and indicated that a decent ratio of genomic loci implicated in psoriasis which could directly affect non-immune cells for immune regulation.

IFN- $\alpha$ could strongly activate immature DCs to produce IL-12, IL-15, IL-18, and IL-23, which acted as upstream cytokines along the IL-23/IL-17 axis to initiate psoriatic inflammation ${ }^{52}$. However, a clinical trial of an anti-IFN- $\alpha$ agent did not show clinical improvement in patients with psoriasis $^{53}$. Our GO analysis showed that upregulated genes of skin resident cell subpopulations in psoriasis vulgaris were abundantly enriched in type I interferon-related functions, which was consistent with the previous result, suggested a crucial role in the disease. However, many immune GO-bioprocesses other than interferon-related functions were enriched from upregulated genes of psoriatic skin resident cells, as shown in (Fig. 3E, F, SI Fig. 3). Importantly, these immune functions could not be dispelled completely by INF- $\alpha$ or frequently-used TNF- $\alpha$, IL-17, 
IL-23 targeted therapies. With these enriched alternative immune functions, the upregulated genes in the psoriatic skin resident cells further amplified the inflammatory response, which led to the persistence of localized inflammation, thus caused an alternative hit to patients.

Interestingly, the skin resident cells such as epidermal basal cells, hair follicle-like cells, mesenchymal cells, dermal papilla-like cells, and Schwann cells significantly evoked MHC complex molecules in psoriatic condition. MHC molecules can modulate various types of immune cells via targeting CD4, CD8, CD94 (ref. ${ }^{54-56}$ ). And the conditional expression of MHC molecules has been observed in the keratinocytes, dermal fibroblasts, and oligodendrocytes $^{57-59}$. Therefore, our findings that the expression of MHC molecules in psoriatic skin resident cells induced by psoriasis provided a novel insight of the immune regulation from skin resident cells to infiltrated immune cells. Perturbation of MHC related genes has been used for establishing animal model of models of psoriasis $^{60-63}$. The increased expression of MHC-related molecules in human local resident skin cells, especially, HLA-C, may be crucial for transforming the acute inflammation into a chronic disease ${ }^{64}$, which is possibly a key element for building the mouse model of psoriasis.

Ultimately, we identified the upregulated ligand genes of resident epidermal and mesenchymal cells, including many cytokines like LIF, IL-6, IL-17B, IL-36 and CD58. LIF/IL- 6 has been identified to sustain the fibroblast production of inflammatory mediators ${ }^{42}$. IL-36 was reported as a molecular linker between keratinocytes and $\mathrm{DCs}^{65}$. CD58 was the ligand of CD2 in T cells and NK cells $^{66}$. Here, for the first time, we observed that IL-17B expression increased in the mesenchymal cells in the skin dermis region, possibly upon TNF- $\alpha$ stimulation during psoriasis, which could subsequently trigger DCs activation and potentially other immune cells via binding to its receptor IL17-RB. Therefore, the ligand-receptor linking further demonstrated that local skin resident cells could directly modulate their surrounding immune cells.

In summary, our single cell transcriptional zonation of human full-thickness skin provided a unique insight on the cell-type orchestra in both healthy and psoriatic condition, uncovered the evolutional conserved/specific skin cell types, and systematically proposed the alternative immune-regulatory axis from local resident cells to immune cells. Above all, our findings provided a promising novel target for psoriasis treatment.

\section{Materials and methods}

Tissue isolation and preparation of single cell suspension

Three patients with definite diagnosis of psoriasis vulgaris were selected as donors, and the full-thickness skins of the lesion were excised surgically. Three healthy donors without systemic diseases undergoing surgery were selected as controls, whose normal skins around the surgical margin were taken for the study. Sample digestion was performed using the full-thickness skin dissociation kit for human (Miltenyi Biotec, Germany). Enzymatic digestion was completed in $2 \mathrm{~h}$, followed by mechanical dissociation using gentleMacs Dissociator (Miltenyi Biotec), running the gentleMACS program h_skin_01. Single cell suspension with the cell counts more than 500000 and alive cell rate more than $90 \%$ observed under a microscope could be tested in the next step.

\section{Preparation of scRNAseq library, sequencing and data pre- processing}

Single cell cDNA libraries were prepared by using 10X Genomics Chromium Single Cell 3' Reagent Kits (version 3.1) according to the manufacturer's instruction, and the constructed libraries were sequenced on illumina novaseq 6000 with paired end $150 \mathrm{bp}$. Raw sequencing data were processed via the 10X Genomics Cell Ranger (version 3.0.2) by using GRCh38.p12 genome assembly. According to the evaluation of quality control, cells harboring $<500$ transcripts, $<1000$ unique molecules, $>5 \%$ ratio of mitochondria, and predicted doublets were removed.

\section{Cell clustering and marker gene enrichment}

Before clustering, we removed the cell cycle genes and obtained the overdispersed genes by estimating the mean and coefficients of variation. The overdispersed genes were then used for cell clustering via Louvain algorithm for community detection (Seurat V2.0 $+{ }^{67}$. For each main cell type, we further zoomed-in for clustering the sub-clusters. As each cell type with different features, we set the neighboring numbers range between $5-25$, and selected PCs according to the elbow plot (normally top 20 PCs). And the resolution was between $0.8-1.5$. Some clusters were merged or further separated to eventually obtain significant marker genes for each cluster. The significant marker genes of each cluster were determined via Enrichment Score with a cutoff of false positive rate $<0.1$ (ref. ${ }^{68}$ ).

\section{Probabilistic scoring analysis of cell-type identity in neural network model}

To build a classify for scoring the probabilistic cell identity of each cell relative to the defined cell types at the transcriptional level ${ }^{69,70}$, a vanilla neural network model for classification task was constructed in PyTorch framework with Skorch package, and trained the model to interactively learn the transcriptional features of defined cell types. To train the model, we obtained the marker genes of each cell type that previously defined by enrichment score and simultaneously ranked by the expression fold-change. The cross-species alignment was performed as described in the previous study ${ }^{71}$. Data were visualized in the radar-shield plot $^{72}$. 
GO-bioprocess enrichment and ligand-receptor analysis

GO enrichment was performed with custom-built SQL dataset originally from Gene Ontology Resource (www. geneontology.org). Pipeline was modified from goenrich package, highlighting the significant enriched GO terms in red frame and specifically enriching the GO-bioprocess. The multi-layer enrichment plots were visualized via NetworkX. Ligand-receptor analysis were performed via nichenetr package with modification that specifically extract the ligand-to-receptor event, and instead of evaluating the ligand-receptor activity, the ligands were selected via psoriasis upregulated genes of each cell types, and corresponding receptors were extracted with the criteria of constant or upregulated expression. All interactions of each cell type were visualized by chord plot. Ligand-to-receptor score of each cell type-cell type pair was calculated via the ratio between the active interaction event and the total interaction event. The top active interactions were selected once the sorted overall ratio curve of all interactions reaches the edge curve plateaus, thus the cutoff was set as 0.35 . Specific ligand-receptor interactions between two cell types were visualized via Sankey diagrams plot.

\section{Immunofluorescence and immunohistochemistry}

For immunohistochemistry staining, skin tissue sections were incubated with primary antibodies of anti-PDGFRB (YT3639, Immunoway, China), anti-IL-17B (bs-2609R, Bioss, China), and secondary antibodies of HRP-Goat anti rabbit IgG (RS0002, Immunoway), HRP-Goat anti mouse IgG (RS0001, Immunoway). For immunofluorescence, skin tissue sections were incubated with primary antibodies of anti-PDGFRB (ab156762, Abcam, UK), anti-IL17B (bs2609, Bioss), and secondary antibodies of Goat Anti-Rabbit IgG (ab6939, Abcam), Goat Anti Mouse IgG (RS0003, Immunoway). The slides were mounted with Vectashield with DAPI (Vector Laboratories, CA, USA). Laser-scanning confocal images were acquired using the BX51 microscope (Olympus, Japan).

\section{DC activation}

DCs were generated from the bone marrow cells of 8 weeks old female BALB/c mice. The bone marrow cells were centrifuged, washed and cultured in roswell park memorial institute 1640 medium (Hyclone, Utah, USA) containing $10 \%$ fetal bovine serum (Sciencell, CA, USA), $1 \%$ penicillin/streptomycin (Beyotime), $10 \mathrm{ng} / \mathrm{ml} \mathrm{recom-}$ binant human IL-4 protein (R\&D systems, Minnesota, USA) and $20 \mathrm{ng} / \mathrm{ml}$ granulocyte macrophage-colony stimulating factor (PeproTech, NJ, USA) and incubated at $37^{\circ} \mathrm{C}$ and $5 \% \mathrm{CO} 2$ for seven days. Cells were cultured with recombinant human IL-17B protein $(500 \mathrm{ng} / \mathrm{mL})$ (R\&D systems) for $24 \mathrm{~h}$. Cells cultured with medium provided a negative control while cells cultured with LPS
(10 ng/mL) (Sigma, Missouri, USA) provided the DC activation positive control. DC phenotypes were analyzed via surface expression of specific markers. Live DCs were immunolabeled with APC-CY7-livedead (Thermo Fisher Scientific, Massachusetts, USA), efluor450-CD11C (Thermo Fisher Scientific), PE-CD80 (Thermo Fisher Scientific), APC-CD86 (Thermo Fisher Scientific), FITCMHC II (Thermo Fisher Scientific) at $4{ }^{\circ} \mathrm{C}$ for $30 \mathrm{~min}$. Resuspend cells were detected on Beckman Cytoflex LX and the result was analyzed by FlowJo 10 .

\section{PCR-Sequence specific primer of HLA-C*06:02}

Fresh blood $(200 \mu \mathrm{l})$ was collected into EDTA containing tube and DNA was extracted using QIAamp Blood Mini Kit (Qiagen, Germany). PCR was performed according to the manufacturer's recommendations. Primers of human HLA-C*06:02 were F-TACTACAACCAG AGCGAGGA; R-GGTCGCAGCCATACATCCA. PCR products were electrophoresed in $2 \%$ agarose gels containing $0.5 \mathrm{mg} / \mathrm{ml}$ ethidium bromide. The gels were run for $35 \mathrm{~min}$ at $100 \mathrm{~V}$ in TAE buffer, and the gel was scanned.

\section{Primary cell isolation and ELISA for chemokines detection}

Skin samples were placed in $0.25 \%$ dispase II (Roche, Switzerland) solution at $4{ }^{\circ} \mathrm{C}$ for $16 \mathrm{~h}$. The epidermis and dermis were separated by forceps. The epidermis was cut and put into $0.25 \%$ trypsin and $0.02 \%$ EDTA digestion (Beyotime), and the cells were cultured in the defined keratinocyte-SFM (Gibco, New York, USA) for 5 days to get primary keratinocytes. The dermis was cut into small pieces and cultured in Dulbecco's modified eagle medium (Gibco) containing 10\% fetal bovine serum (Sciencell), 1\% penicillin/streptomycin (Beyotime) for 7 days to get primary fibroblast. Cells were cultured with recombinant human TNF- $\alpha$ protein (Abcam) for $12 \mathrm{~h}$, cells cultured with medium provided a control group. Culture supernatants were collected to measure the levels of cytokines secreted by keratinocytes and fibroblast. The levels of CXCL8 (Raybiotech, Georgia, USA), CCL20 (Abcam), IL6 (Invitrogen), IL-1B (Abcam), and IL-17B (Novus Biologicals, Colorado, USA) were determined by ELISA according to the manufacturer's instructions.

\section{Quantification of images and statistical analysis}

Semi-quantification of the staining was performed independently using Image-Pro Plus 6.0. Statistical analysis was performed using GraphPad Prism version 8.0. Data were presented as mean \pm SEM. Statistical significance was assessed by Student's $t$-test.

\section{Acknowledgements}

YG.G. thanks Mart Saarma and Maria Lindahl from Institute of Biotechnology, HiLIFE, University of Helsinki for comments and technical arrangement. YZ.H thanks Patrik Ernfors, Maria Kasper and Karl Annusver from Karolinska Institutet 
for kind supports and critical reading of the manuscript. YZ.H further appreciates the data supports from Jeffrey B Cheng and Raymond J Cho from University of California. We sincerely thank the generous help of Yan Wang from the third affiliated hospital of Harbin Medical University in surgical materials, Novogene Co., Ltd (Beijing, China) for single cell RNA sequencing, PC-biotech Co., Ltd (Shanghai, China) for biochemical supports.

\section{Author details}

'Department of Dermatology, The Second Affiliated Hospital of Harbin Medical University, No.246 Xuefu Road, 150001 Harbin, China. ${ }^{2}$ Institute of Biotechnology, HILIFE Unit, University of Helsinki, 00014 Helsinki, Finland. ${ }^{3}$ Department of Dermatology, Peking University First Hospital, No.8 XiShiKu Street, 100034 Beijing, China. ${ }^{4}$ Department of Oncology, The Second Affiliated Hospital of Harbin Medical University, No.246 Xuefu Road, 150001 Harbin, China. ${ }^{5}$ Division of Molecular Neurobiology, Department of Medical Biochemistry and Biophysics, Karolinska Institutet, Solnavägen 9, 17165 Solna, Stockholm, Sweden

\section{Author contributions}

Y.Z.L. and Y.Z.H. conceived and designed the study, Y.Z.H. supervised and performed the data analysis. Y.G.G. performed the experiments and analysis, $Y$. Z.L.,Y.Z.H., Y.G.G. performed development of methodology and writing of the paper, X.Y.Y., Y.M.Z., H.N.L.L.L., X.Q.S., P.Y. and T.K.X. provided biopsies acquisition, data analysis and, and statistical analysis. Y.Z.L.,Y.Z.H., Y.G.G., X.Y.Y., Y.M.Z. made the interpretation of data.

\section{Funding}

This work is supported by grants from the National Natural Science Foundation of China (81972925), the National Natural Science Foundation of China (81702464) and the Swedish Society for Medical Research Scholarship. The funders had no role in study design, data collection and analysis, decision to publish, or preparation of the manuscript.

\section{Data availability}

The processed sequencing data reported in this paper is in Gene Expression Omnibus (GEO) database: GSE162183. Raw data in this paper will be deposited in European Genome-Phenome Archive (EGA). Webpage of data visualization: https://yzstudio.one/skin-psoriasis-atlas. Public data used in this study were downloaded from EGA: EGAS00001002927; GEO: GSE130973, GSE150361 \& GSE129218. Code details are available upon reasonable request.

\section{Ethics statement}

The research was obtained by the Second Affiliated Hospital of Harbin Medical University Medical Ethics Committee approved. All patients provided written informed consent.

\section{Conflict of interest}

The authors declare no competing interests.

\section{Publisher's note}

Springer Nature remains neutral with regard to jurisdictional claims in published maps and institutional affiliations.

Supplementary information The online version contains supplementary material available at https://doi.org/10.1038/s41419-021-03724-6.

Received: 30 November 2020 Revised: 13 April 2021 Accepted: 14 April 2021

Published online: 06 May 2021

\section{References}

1. Parisi, R. et al. Global epidemiology of psoriasis: a systematic review of incidence and prevalence. J. Investigative Dermatol. 133, 377-385 (2013).

2. Boehncke, W. H. \& Schon, M. P. Psoriasis. Lancet 386, 983-994 (2015).

3. Chiricozzi, A., Romanelli, P., Volpe, E., Borsellino, G. \& Romanelli, M. Scanning the Immunopathogenesis of Psoriasis. Int. J. Mol. Sci. 19, 179 (2018).

4. Greb, J. E. et al. Psoriasis. Nat. Rev. Dis. Prim. 2, 16082 (2016).
}

5. Nestle, F. O., Kaplan, D. H. \& Barker, J. Psoriasis. N. Engl. J. Med. 361, 496-509 (2009).

6. Boehncke, W. H. Systemic inflammation and cardiovascular comorbidity in psoriasis patients: causes and consequences. Front. Immunol. 9, 579 (2018).

7. Conrad, C. \& Gilliet, M. Psoriasis: from pathogenesis to targeted therapies. Clin. Rev. Allergy Immunol. 54, 102-113 (2018).

8. Chan, T. C., Hawkes, J. E. \& Krueger, J. G. Interleukin 23 in the skin: role in psoriasis pathogenesis and selective interleukin 23 blockade as treatment. Therapeutic Adv. Chronic Dis. 9, 111-119 (2018).

9. Krueger, J. G. et al. IL-17A is essential for cell activation and inflammatory gene circuits in subjects with psoriasis. J. Allergy Clin. Immunol. 130, 145-154 e149 (2012).

10. Fotiadou, C. et al. IL-17A, IL-22, and IL-23 as markers of psoriasis activity: a cross-sectional, hospital-based study. J. Cutan. Med. Surg. 19, 555-560 (2015).

11. Di Cesare, A., Di Meglio, P. \& Nestle, F. O. The IL-23/Th17 axis in the immunopathogenesis of psoriasis. J. Investigative Dermatol. 129, 1339-1350 (2009).

12. Gisondi, P., Geat, D., Pizzolato, M. \& Girolomoni, G. State of the art and pharmacological pipeline of biologics for chronic plaque psoriasis. Curr. Opin. Pharmacol. 46, 90-99 (2019).

13. Kabashima, K., Honda, T., Ginhoux, F. \& Egawa, G. The immunological anatomy of the skin. Nat. Rev. Immunol. 19, 19-30 (2019).

14. $\mathrm{He}, \mathrm{H}$. et al. Single-cell transcriptome analysis of human skin identifies novel fibroblast subpopulation and enrichment of immune subsets in atopic dermatitis. J. Allergy Clin. Immunol. 145, 1615-1628 (2020).

15. A R, Yu, P., Hao, S. \& Li, Y. MiR-876-5p suppresses cell proliferation by targeting Angiopoietin-1 in the psoriasis. Biomedicine Pharmacother. = Biomedecine Pharmacotherapie 103, 1163-1169 (2018).

16. Abu Tayeh, A. et al. Psoriasis-like skin disorder in transgenic mice expressing a RIG-I Singleton-Merten syndrome variant. Int. immunol. 33, 211-224 (2020).

17. Moldovan, L. I. et al. High-throughput RNA sequencing from paired lesionaland non-lesional skin reveals major alterations in the psoriasis circRNAome. BMC Med. Genomics 12, 174 (2019).

18. Dand, N. et al. HLA-C*06:02 genotype is a predictive biomarker of biologic treatment response in psoriasis. J. Allergy Clin. Immunol. 143, 2120-2130 (2019).

19. Prinz, J. C. Human leukocyte antigen-class I alleles and the autoreactive T cell response in psoriasis pathogenesis. Front. Immunol. 9, 954 (2018).

20. DeWard, A. D., Cramer, J. \& Lagasse, E. Cellular heterogeneity in the mouse esophagus implicates the presence of a nonquiescent epithelial stem cell population. Cell Rep. 9, 701-711 (2014).

21. Moll, R., Divo, M. \& Langbein, L. The human keratins: biology and pathology. Histochemistry Cell Biol. 129, 705-733 (2008).

22. Reveiller, M. et al. Bile exposure inhibits expression of squamous differentiation genes in human esophageal epithelial cells. Ann. Surg. 255, 1113-1120 (2012).

23. Sumer, C., Boz, E. R. A. B. \& Dincer, T. Keratin 14 is a novel interaction partner of keratinocyte differentiation regulator: receptor-interacting protein kinase 4. Turkish J. Biol. = Turk. Biyol. Derg. 43, 225-234 (2019).

24. Shih, B. B. et al. Derivation of marker gene signatures from human skin and their use in the interpretation of the transcriptional changes associated with dermatological disorders. J. Pathol. 241, 600-613 (2017).

25. Rittie, L., Stoll, S. W., Kang, S., Voorhees, J. J. \& Fisher, G. J. Hedgehog signaling maintains hair follicle stem cell phenotype in young and aged human skin. Aging Cell 8, 738-751 (2009).

26. Mardaryev, A. N. et al. Lhx2 differentially regulates Sox9, Tcf4 and Lgr5 in hair follicle stem cells to promote epidermal regeneration after injury. Development 138, 4843-4852 (2011).

27. Sabbagh, M. F. et al. Transcriptional and epigenomic landscapes of CNS and non-CNS vascular endothelial cells. elife 7, e36187 (2018).

28. Vandamme, N. \& Berx, G. From neural crest cells to melanocytes: cellular plasticity during development and beyond. Cell. Mol. Life Sci.: CMLS 76, 1919-1934 (2019)

29. Guerrero-Juarez, C. F. et al. Single-cell analysis reveals fibroblast heterogeneity and myeloid-derived adipocyte progenitors in murine skin wounds. Nat. Commun. 10, 650 (2019).

30. Joost, S. et al. The molecular anatomy of mouse skin during hair growth and rest. Cell Stem Cell 26, 441-457 e447 (2020).

31. Cheng, J. B. et al. Transcriptional programming of normal and inflamed human epidermis at single-cell resolution. Cell Rep. 25, 871-883 (2018).

32. Sole-Boldo, L. et al. Single-cell transcriptomes of the human skin reveal agerelated loss of fibroblast priming. Commun. Biol. 3, 188 (2020).

33. Fang, D. et al. Defining the conditions for the generation of melanocytes from human embryonic stem cells. Stem Cells 24, 1668-1677 (2006). 
34. Etxaniz, U. et al. Neural-competent cells of adult human dermis belong to the Schwann lineage. Stem Cell Rep. 3, 774-788 (2014).

35. Bray, E. R., Cheret, J., Yosipovitch, G. \& Paus, R. Schwann cells as underestimated, major players in human skin physiology and pathology. Exp. Dermatol. 29, 93-101 (2020)

36. Lou, F. et al. Excessive polyamine generation in keratinocytes promotes selfRNA sensing by dendritic cells in psoriasis. Immunity 53, 204-216 e210 (2020).

37. Tsoi, L. C. et al. Identification of 15 new psoriasis susceptibility loci highlights the role of innate immunity. Nat. Genet. 44, 1341-1348 (2012).

38. Zuo, X. et al. Whole-exome SNP array identifies 15 new susceptibility loci for psoriasis. Nat. Commun. 6, 6793 (2015).

39. Gresnigt, M. S. \& van de Veerdonk, F. L. Biology of IL-36 cytokines and their role in disease. Semin. Immunol. 25, 458-465 (2013).

40. Valencia, J. et al. Wnt5a signaling increases IL-12 secretion by human dendritic cells and enhances IFN-gamma production by CD4+ T cells. Immunol. Lett. 162, 188-199 (2014).

41. Leitner, J., Herndler-Brandstetter, D., Zlabinger, G. J., Grubeck-Loebenstein, B. \& Steinberger, P. CD58/CD2 is the primary costimulatory pathway in human CD28-CD8+ T cells. J. Immunol. 195, 477-487 (2015)

42. Nguyen, H. N. et al. Autocrine loop involving IL-6 family member LIF, LIF receptor, and STAT4 drives sustained fibroblast production of inflammatory mediators. Immunity 46, 220-232 (2017)

43. Li, S. et al. Defining key genes regulating morphogenesis of apocrine sweat gland in sheepskin. Front. Genet. 9, 739 (2018).

44. Reynolds, G. et al. Developmental cell programs are co-opted in inflammatory skin disease. Science 371, 6527 (2021)

45. Liu J. et al. Single-cell RNA sequencing of psoriatic skin identifies pathogenic Tc17 cell subsets and reveals distinctions between CD8(+) T cells in autoimmunity and cancer. J. Allergy Clin. Immunol. (2020).

46. Qie, C. et al. Single-cell RNA-Seq reveals the transcriptional landscape and heterogeneity of skin macrophages in Vsir(-/-) murine psoriasis. Theranostics 10, 10483-10497 (2020).

47. Joost, S. et al. Single-Cell transcriptomics reveals that differentiation and spatial signatures shape epidermal and hair follicle heterogeneity. Cell Syst. 3, 221-237 e229 (2016).

48. Bandari, A. K. et al. A novel splice site mutation in IFNGR2 in patients with primary immunodeficiency exhibiting susceptibility to mycobacterial diseases. Front. Immunol. 10, 1964 (2019).

49. Piskin, G., Sylva-Steenland, R. M., Bos, J. D. \& Teunissen, M. B. In vitro and in situ expression of IL-23 by keratinocytes in healthy skin and psoriasis lesions: enhanced expression in psoriatic skin. J. Immunol. 176, 1908-1915 (2006).

50. Nakajima, K. et al. Stat3 activation in epidermal keratinocytes induces Langerhans cell activation to form an essential circuit for psoriasis via IL-23 production. J. Dermatological Sci. 93, 82-91 (2019).

51. Devos, M. et al. Keratinocyte expression of A20/TNFAIP3 controls skin inflammation associated with atopic dermatitis and psoriasis. J. Investigative Dermatol. 139, 135-145 (2019).

52. Lande, R. \& Gilliet, M. Plasmacytoid dendritic cells: key players in the initiation and regulation of immune responses. Ann. N. Y. Acad. Sci. 1183, 89-103 (2010).
53. Bissonnette, R. et al. A randomized, double-blind, placebo-controlled, phase I study of MEDI-545, an anti-interferon-alfa monoclonal antibody, in subjects with chronic psoriasis. J. Am. Acad. Dermatol. 62, 427-436 (2010).

54. Fleury, S. et al. HLA-DR polymorphism affects the interaction with CD4. J. Exp. Med. 182, 733-741 (1995).

55. Sun, J., Leahy, D. J. \& Kavathas, P. B. Interaction between CD8 and major histocompatibility complex (MHC) class I mediated by multiple contact surfaces that include the alpha 2 and alpha 3 domains of MHC class I. J. Exp. Med. 182, 1275-1280 (1995).

56. Lee, N. et al. HLA-E is a major ligand for the natural killer inhibitory receptor CD94/NKG2A. Proc. Natl Acad. Sci. USA 95, 5199-5204 (1998).

57. Umetsu, D. T., Katzen, D., Jabara, H. H. \& Geha, R. S. Antigen presentation by human dermal fibroblasts: activation of resting $\mathrm{T}$ lymphocytes. J. Immunol. 136, 440-445 (1986).

58. Fan, L. et al. Antigen presentation by keratinocytes directs autoimmune skin disease. Proc. Natl Acad. Sci. USA 100, 3386-3391 (2003).

59. Falcao, A. M. et al. Disease-specific oligodendrocyte lineage cells arise in multiple sclerosis. Nat. Med. 24, 1837-1844 (2018).

60. Wagner, E. F., Schonthaler, H. B., Guinea-Viniegra, J. \& Tschachler, E. Psoriasis: what we have learned from mouse models. Nat. Rev. Rheumatol. 6, 704-714 (2010).

61. Weitz, J. E. \& Ritchlin, C. T. Mechanistic insights from animal models of psoriasis and psoriatic arthritis. Curr. Rheumatol. Rep. 15, 377 (2013).

62. Khare, S. D., Hansen, J., Luthra, H. S. \& David, C. S. HLA-B27 heavy chains contribute to spontaneous inflammatory disease in B27/human beta2microglobulin (beta2m) double transgenic mice with disrupted mouse beta2m. J. Clin. Investig. 98, 2746-2755 (1996).

63. Schon, M. P., Detmar, M. \& Parker, C. M. Murine psoriasis-like disorder induced by naive CD4+ T cells. Nat. Med. 3, 183-188 (1997).

64. van den Bogaard, E. H. et al. Cell surface expression of HLA-Cw6 by human epidermal keratinocytes: positive regulation by cytokines, lack of correlation to a variant upstream of HLA-C. J. Investigative Dermatol. 136, 1903-1906 (2016).

65. Tortola, L. et al. Psoriasiform dermatitis is driven by IL-36-mediated DCkeratinocyte crosstalk. J. Clin. Investig. 122, 3965-3976 (2012).

66. Shao, $\mathrm{T}$. et al. Costimulatory function of $\mathrm{Cd} 58 / \mathrm{Cd} 2$ interaction in adaptive humoral immunity in a zebrafish model. Front. Immunol. 9, 1204 (2018).

67. Stuart, T. \& Satija, R. Integrative single-cell analysis. Nat. Rev. Genet. 20, 257-272 (2019).

68. Zeisel, A. et al. Molecular architecture of the mouse nervous system. Cell 174, 999-1014 e1022 (2018).

69. Capper, D. et al. DNA methylation-based classification of central nervous system tumours. Nature 555, 469-474 (2018).

70. Malta, T. M. et al. Machine learning identifies stemness features associated with oncogenic dedifferentiation. Cell 173, 338-354 e315 (2018).

71. La Manno, G. et al. Molecular diversity of midbrain development in Mouse, Human, and stem. Cells Cell 167, 566-580.e519 (2016).

72. Hovestadt, V. et al. Resolving medulloblastoma cellular architecture by singlecell genomics. Nature 572, 74-79 (2019). 